\title{
S? \\ Clausius-Clapeyron relations for first-order phase transitions in bilayer quantum Hall systems
}

\author{
Yue Zou (邹悦), ${ }^{1}$ Gil Refael, ${ }^{1}$ Ady Stern, ${ }^{2}$ and J. P. Eisenstein ${ }^{1}$ \\ ${ }^{1}$ Department of Physics, California Institute of Technology, Pasadena, California 91125, USA \\ ${ }^{2}$ Department of Condensed Matter Physics, Weizmann Institute of Science, Rehovot 76100, Israel \\ (Received 26 December 2009; revised manuscript received 28 April 2010; published 17 May 2010)
}

\begin{abstract}
A bilayer system of two-dimensional electron gases in a perpendicular magnetic field exhibits rich phenomena. At total filling factor $\nu_{t o t}=1$, as one increases the layer separation, the bilayer system goes from an interlayer-coherent exciton condensed state to an incoherent phase of, most likely, two decoupled compositefermion Fermi liquids. Many questions still remain as to the nature of the transition between these two phases. Recent experiments have demonstrated that spin plays an important role in this transition. Assuming that there is a direct first-order transition between the spin-polarized interlayer-coherent quantum Hall state and spin partially polarized composite Fermi-liquid state, we calculate the phase boundary $(d / l)_{c}$ as a function of parallel magnetic field, NMR/heat pulse, temperature, and density imbalance, and compare with experimental results. Remarkably good agreement is found between theory and various experiments.
\end{abstract}

DOI: $10.1103 /$ PhysRevB.81.205313

\section{INTRODUCTION}

A bilayer two-dimensional electron gas (2DEG) in a strong perpendicular magnetic field at total filling factor $\nu_{t o t}=1$ exhibits rich phenomena. An important tuning parameter in this system is the ratio $d / l$, where $d$ is the effective interlayer distance and $l$ is the magnetic length. At small $d / l$, even with negligible tunneling, a remarkable bilayer quantum Hall state with interlayer phase coherence emerges due to interlayer Coulomb interaction. This bilayer quantum Hall state can be described as a pseudospin ferromagnet where the layer index acts as the pseudospin or an exciton condensate formed by interlayer particle-hole pairing. ${ }^{1-3}$ Many remarkable experimental signatures of this phase predicted by theories have been observed in experiments, including enormous enhancement of zero-bias interlayer tunneling, ${ }^{4}$ linearly dispersing Goldstone mode, ${ }^{5}$ quantized Hall drag, ${ }^{6}$ and vanishing resistance in counterflow. ${ }^{7}$ However, there are still important discrepancies between theory and experiment. For example, the height of the interlayer tunneling conductance is observed to be finite ${ }^{4}$ while theories predict it to be infinite. Also, transport in counterflow experiments should be completely dissipationless under a critical temperature for phase coherence but in experiments dissipationless counterflow is only seen in the zero-temperature limit. ${ }^{7}$ The effect of quenched disorder is believed to be crucial to reconcile these discrepancies, ${ }^{8-10}$ although a quantitative understanding is still lacking.

The nature of the phase transition when $d / l$ is increased and the quantum Hall phase is destroyed is even more puzzling. In the limit $d / l \rightarrow \infty$, each layer is at half filling, and they should behave as weakly coupled composite Fermi liquids. Much progress have been made in understanding this phase using the Chern-Simons approach ${ }^{11-15}$ and the dipolar quasiparticle approach. ${ }^{16-21}$ Although we understand well both the coherent phase at $d / l \rightarrow 0$ and the composite Fermi-liquid state at $d / l \rightarrow \infty$, the transition between them has been shrouded in mystery. There have been many experimental $^{22-35}$ and theoretical ${ }^{36-48}$ studies regarding the nature of this transition. While some of these theoretical works point to a direct transition between the two limiting
PACS number(s): 73.43.Nq, 71.35.Lk, 71.10.Pm

phases, either continuous ${ }^{45}$ or of first order, ${ }^{42,43}$ some other works predict the existence of various types of exotic intermediate phases, including translational symmetry broken phase, ${ }^{36-38,46}$ composite-fermion paired state, ${ }^{39,40,47}$ phase of coexisting composite fermions and composite bosons, ${ }^{44,48,49}$ quantum disordered phases, ${ }^{41}$ etc.

These theoretical works typically assume that the physical spin is fully polarized and hence irrelevant across the transition. However, recent experiments have shown that spin plays an important role in the transition. Reference 26 has found that by applying a NMR pulse or heat pulse to depolarize the nuclei and hence increasing the effective magnetic field coupled to the spin, the coherent phase is strengthened, and the phase boundary shifts to higher value of $d / l$. Similar behavior has also been observed by applying a parallel magnetic field. ${ }^{30}$ These experimental results indicate that at least one of the phases involved in the transition is not fully polarized and that the polarization changes significantly across the transition. The most likely possibility is that the incoherent composite Fermi-liquid phase at large $d / l$ is only partially polarized, as shown by other experiments on single layer at $\nu=1 / 2 .{ }^{50,51}$ If the transition between the coherent phase and the less polarized incoherent phase is a thermodynamic phase transition, it must be of first order: the magnetization is discontinuous across the transition, and, as the experiments of Ref. 26 found, the transition can be tuned using a Zeeman field which is conjugate to the magnetization. These two facts together imply the first-order nature of the transition. An alternative to the thermodynamic transition scenario is a singularity-free quantum crossover as was suggested recently in Refs. 47 and 48.

In this work, we assume that the transition tuned by $d / l$ is a thermodynamic first-order transition between spinpolarized coherent $\nu_{t o t}=1$ quantum Hall state and partially polarized composite Fermi-liquid state, and derive the Clausius-Clapeyron relations for this system. The ClausiusClapeyron relations will allow us to obtain the phase boundary shapes for the transition; a comparison of these boundaries with experiments presents a stringent consistency test of the first-order transition scenario. The first-order scenario was invoked by Ref. 43 to explain the strongly enhanced 
longitudinal Coulomb drag for intermediate $d / l$, and it also has some support from exact-diagonalization study. ${ }^{42}$ Note that we will only consider the case of negligible interlayer tunneling.

The Clausius-Clapeyron relations are the results of matching the free energies of the two phases along the phase boundary. To be more specific, we denote the free-energy density of the coherent and the incoherent phases to be $E_{c}$ and $E_{i}$, and define

$$
f\left(\delta, B_{t o t}, \Delta n, T\right)=E_{c}\left(\delta, B_{t o t}, \Delta n, T\right)-E_{i}\left(\delta, B_{t o t}, \Delta n, T\right),
$$

where $\delta \equiv d / l, B_{\text {tot }}$ is the total magnetic field coupled to electrons' physical spin, $\Delta n=\left(n_{1}-n_{2}\right) / 2$ is the density imbalance, and $T$ is the temperature. At any point along the phase boundary, we must have

$$
f\left(\delta_{c}, B_{t o t}, \Delta n, T\right)=0 .
$$

This equation can be viewed as defining the $\delta_{c}$ at which the transition occurs. When one changes the total field by $d B_{t o t}$, the critical $\delta_{c}\left(B_{t o t}, \Delta n, T\right)$ also changes by $d \delta_{c}$ when the filling factor is kept fixed at $\nu_{t o t}=1$. Their relation is determined by

$$
0=\frac{\partial f}{\partial \delta} d \delta_{c}+\frac{\partial f}{\partial B_{t o t}} d B_{t o t}
$$

therefore, the slope of the phase boundary is determined by the following ordinary differential equation (ODE),

$$
\frac{d \delta_{c}}{d B_{t o t}}=-\frac{\frac{\partial f}{\partial B_{t o t}}}{\frac{\partial f}{\partial \delta}}=\frac{\frac{\partial E_{i}}{\partial B_{t o t}}-\frac{\partial E_{c}}{\partial B_{t o t}}}{\frac{\partial f}{\partial \delta}}
$$

A crucial assumption of our work is that

$$
\frac{\partial f}{\partial \delta}=\eta \frac{e^{2}}{\epsilon l^{3}},
$$

where $e^{2} /\left(\epsilon l^{3}\right)$ not only gives the correct units but is the only energy scale that exists in this problem if we neglect the Landau-level mixing. $\eta$ is a universal positive dimensionless constant. It is positive because $f$ should be an increasing function of $\delta=d / l$ since the incoherent phase should be more and more energetically favorable with increasing $d / l$. In general, $\eta$ could be a function of $\delta=d / l$, i.e., $\eta(\delta) \approx \eta\left(\delta_{0}\right)$ $+\mathcal{O}\left[\left(\delta-\delta_{0}\right) / \delta_{0}\right]$, but since in experiments $\delta$ does not change much (ranging from 1.7 to 2 ), $\left(\delta-\delta_{0}\right) / \delta_{0} \ll 1$, we will assume $\eta$ to be a constant for simplicity.

Similar analysis also applies to finite-temperature transitions,

$$
\frac{d \delta_{c}}{d T}=\frac{\frac{\partial E_{i}}{\partial T}-\frac{\partial E_{c}}{\partial T}}{\eta \frac{e^{2}}{\epsilon l^{3}}}
$$

For density imbalance experiments, we will focus on the phase boundary near $\Delta n=0$. First, note that by symmetry

$$
\frac{\partial f}{\partial \Delta n}=0
$$

Thus, we need to expand $f$ to second order in $\Delta n$,

$$
0=\frac{\partial f}{\partial \delta} d \delta_{c}+\frac{1}{2} \frac{\partial^{2} f}{\partial \Delta n^{2}}(\Delta n)^{2}
$$

and, therefore,

$$
\frac{d \delta_{c}}{d\left(\Delta n^{2}\right)}=\frac{\frac{1}{2} \frac{\partial^{2} E_{i}}{\partial \Delta n^{2}}-\frac{1}{2} \frac{\partial^{2} E_{c}}{\partial \Delta n^{2}}}{\eta \frac{e^{2}}{\epsilon l^{3}}} .
$$

The above equations constitute the Clausius-Clapeyron relations for the bilayer quantum Hall systems. In the following sections, we will investigate whether the phase boundary shapes implied by Clausius-Clapeyron relations are consistent with experiments, and whether a single universal parameter $\eta$ can explain all available experimental results. To obtain the detailed forms of free energy of both phases, we will primarily work with the pseudospin ferromagnet description for the coherent quantum Hall phase and the Chern-Simons approach for the incoherent composite Fermi-liquid phase. Spin transitions, finite-temperature transitions, and density imbalance experiments are studied in Secs. II-IV, respectively. Finally, we summarize and discuss our results in Sec. V. Some theoretical details are relegated to Appendices A and $\mathrm{B}$.

\section{SPIN TRANSITION EXPERIMENTS}

References 26 and 30 have studied the effect of NMR/ heat pulse and parallel magnetic field on the transition tuned by $d / l$, respectively. In the experiment of Ref. 30, since the interlayer tunneling is negligible, the main effect of the parallel field is on the spins of electrons. Similarly, in the experiment of Ref. 26, NMR/heat pulse acts to depolarize the nuclei and therefore also changes the Zeeman field on the electrons through the hyperfine coupling. Thus, these two experiments can be analyzed in a similar fashion. Since we assume the coherent phase is spin polarized, the spin part of the coherent-phase free energy is simply the Zeeman energy,

$$
E_{c}=-\frac{1}{2} N_{T}|g| \mu_{B} B_{t o t}=-\frac{e|g| \mu_{B} B_{\perp} B_{t o t}}{4 \pi \hbar},
$$

where $N_{T}$ is the total electron density of the two layers, $B_{\perp}$ is the perpendicular magnetic field, $B_{t o t}$ is the total magnetic field coupled to electron spin, $g=-0.44$ is the $g$ factor of the GaAs two-dimensional electron gas, and $\mu_{B}$ is the Bohr magneton.

For the partially spin-polarized incoherent phase, the single-layer free energy is

$$
\frac{E_{i}}{2}=\frac{1}{2 \chi} M^{2}-M B_{t o t},
$$

where the magnetization 


$$
M=\frac{1}{2}|g| \mu_{B}\left(n_{\uparrow}-n_{\downarrow}\right) \equiv|g| \mu_{B} \Delta n
$$

and $\chi$ is the single-layer spin susceptibility. The steady state is obtained by minimizing $E_{i}$ with respect to $M$,

$$
\chi=\frac{M}{B_{\text {tot }}},
$$

therefore,

$$
\frac{E_{i}}{2}= \begin{cases}-\frac{1}{2} \chi B_{t o t}^{2}, & B_{t o t}<B_{t o t, p} \\ \frac{1}{2 \chi} M_{\max }^{2}-M_{\max } B_{t o t}, & B_{t o t}>B_{t o t, p},\end{cases}
$$

where the maximum magnetization $M_{\max }$ and the field for full polarization $B_{t o t, p}$ are given by

$$
\begin{gathered}
M_{\max }=\frac{1}{2}|g| \mu_{B} n=\frac{e|g| \mu_{B} B_{\perp}}{8 \pi \hbar}, \\
B_{\text {tot }, p}=\frac{M_{\max }}{\chi} .
\end{gathered}
$$

Plugging these forms of free energy into Eq. (3), we obtain an equation

$$
\frac{d \delta_{c}}{d B_{t o t}}= \begin{cases}\frac{-2 \chi B_{t o t}+\frac{e|g| \mu_{B} B_{\perp}}{4 \pi \hbar}}{\eta \frac{e^{2}}{\epsilon l^{3}}} & B_{t o t}<B_{t o t, p} \\ 0 & B_{t o t}>B_{t o t, p} .\end{cases}
$$

Note that the right-hand side (RHS) also depends on $\delta_{c}$ through $B_{\perp}$ which determines $\ell$. Equation (15) can be solved numerically to yield the $\delta_{c}-B_{t o t}$ curve. For typical experimental parameters, $d \delta_{c} / d B_{t o t}$ starts out to be positive when $B_{\text {tot }}$ is small, and continuously decreases to zero when

$$
-2 \chi B_{\text {tot }}+\frac{e|g| \mu_{B} B_{\perp}}{4 \pi \hbar}=0,
$$

this is nothing but Eq. (14) which determines the magnetic field at which all composite fermions get polarized.

It remains to determine the value of the compositefermion spin susceptibility $\chi$. This can be done if $B_{t o t}$ and $B_{\perp}$ at which full polarization occurs are known because from Eq. (14) or (16) we have

$$
\chi=\frac{|g| \mu_{B} B_{\perp, p}}{4 B_{t o t, p} \phi_{0}},
$$

where the subscript $p$ denotes the point of full polarization. In experimental and exact-diagonalization studies, one often parametrize $\chi$ with the form of noninteracting Fermi gas with a "polarization mass" $m_{p}$ (Refs. 20 and 52),

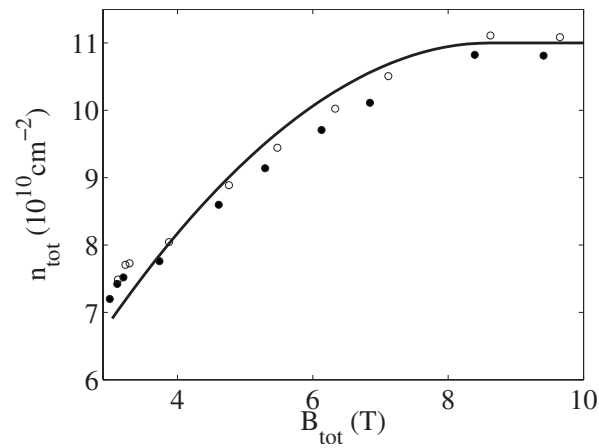

FIG. 1. Total electron density deduced from the critical $d / l$ $=\delta_{c}$ vs the total magnetic field for the parallel magnetic field experiments. Open and solid circles are experimental results of Giudici et al. (Ref. 30) [cf. Fig. 4(a) there]. Solid line is our theoretical calculation with the fitting parameter $\eta=0.8 \times 10^{-3}$. The boundary condition in our calculation is chosen as $n_{t o t}=11 \times 10^{10} \mathrm{~cm}^{-2}$ when $B=10 \mathrm{~T}$.

$$
\chi=\frac{m_{p}}{4 \pi \hbar^{2}}\left(|g| \mu_{B}\right)^{2} .
$$

In the lowest-Landau-level approximation, $\frac{e^{2}}{\epsilon l}$ is the only relevant energy scale and thus

$$
\frac{\hbar^{2}}{l^{2} m_{p}} \propto \frac{e^{2}}{\epsilon l} .
$$

Therefore, presumably $m_{p}$ scales as $\sqrt{B_{\perp}}$,

$$
m_{p}=x m_{e} \sqrt{B_{\perp}},
$$

where $m_{e}$ is the vacuum electron mass, $x$ is a dimensionless number, $B_{\perp}$ is in units of tesla. It is worth noting that unlike free electrons spin susceptibility which is proportional to $1 / m_{e}$, the susceptibility of composite fermions is proportional to $m_{p}$ and therefore to $\sqrt{B}$. The reason for this is that the Bohr magneton $\mu_{B}$ depends on the bare mass of the electron, and therefore does not overturn the proportionality to effective mass in the density-of states factor of the susceptibility.

For the parallel field experiment of Ref. 30, composite fermions get polarized at total density $n_{t o t}=11 \times 10^{10} \mathrm{~cm}^{-2}$, tilting angle $\theta=58^{\circ}$, which corresponds to $B_{\text {tot }, p}=8.60 \mathrm{~T}$, $B_{\perp, p}=4.56 \mathrm{~T}, x=0.56$ if we parametrize $\chi$ in terms of the polarization mass $m_{p}$. Then we solve the ODE, Eq. (15), with the boundary condition at the high-field end point $\left(B_{t o t}\right.$ $=10 \mathrm{~T}, n_{t o t}=11 \times 10^{10} \mathrm{~cm}^{-2}$ ), and plot the $n_{t o t}$ deduced from $\delta_{c}$ vs $B_{\text {tot }}$ in Fig. 1. To tune the result to resemble the experimental results in Fig. 4a of Ref. 30, we get

$$
\eta=(0.8 \pm 0.2) \times 10^{-3},
$$

where the error mainly comes from fitting errors, meaning a finite range of $\eta$ 's make the $\delta_{c}-B_{\text {tot }}$ curve resemble the experimental result.

For the NMR and heat-pulse experiments of Ref. 26, the phase boundary before any perturbation is $\delta_{c 0}=1.967$, which correspond to $B_{\perp}=3.26 \mathrm{~T}$. Reference 26 has estimated the effective nuclear magnetic field to be $B_{N}=-0.17 \mathrm{~T}$, therefore 
the total effective magnetic field felt by electronic spin is $B_{t o t}=B_{\perp}+B_{N}$. After a heat pulse, nuclear spins are depolarized, and $B_{N}$ is set to zero. $B_{\text {tot }}$ is strengthened to $B_{\perp}$, and the phase boundary changes to $\delta_{c}=1.983$. We can not determine the spin susceptibility or the polarization mass directly from experimental information, and therefore we use the numerical and experimental results from the literature $m_{p}$ $=(0.7 \pm 0.2) m_{e} \sqrt{B_{\perp}}$ with $B$ in units of tesla. ${ }^{50,52-55}$ In this way, we obtain

$$
\eta \approx(1.3 \pm 0.4) \times 10^{-3}
$$

where the error mainly comes from uncertainty in the value of the polarization mass $m_{p}$.

Note that our calculations in this section do not rely on the Chern-Simons description of composite fermions.

\section{FINITE-TEMPERATURE TRANSITION EXPERIMENTS}

Reference 31 has studied the changes in critical $\delta_{c}=d / l$ as a function of the temperature $T$. They found that the phase boundary moves to smaller $d / l$ with higher $T$. When analyzing the temperature dependence of the transition, one needs to include the entropy contributions to the free energy associated with various low-energy excitations for both phases. In the interlayer-coherent quantum Hall phase, the only gapless excitation is the linearly dispersing Goldstone mode, which corresponds to in-plane spin wave in the pseudospin language. Therefore, this mode dominates the temperature dependence of the free energy of the coherent phase (see Ref. 56). Denoting its velocity to be $v$, we have the free energy

$$
E_{c}(T)=\sum_{k} T \ln \left(1-e^{-\hbar v k / T}\right) \approx \frac{-1.2}{2 \pi} \frac{T^{3}}{(\hbar v)^{2}}
$$

and, therefore,

$$
\frac{\partial E_{c}}{\partial T}=-\frac{1.8}{\pi} \frac{T^{2}}{(\hbar v)^{2}}
$$

We use the experimental result of Ref. 5 to estimate the value of $v$ (which we assume to be a constant independent of $\delta$ ),

$$
v=1.4 \times 10^{4} \mathrm{~m} \mathrm{~s}^{-1} \text {. }
$$

For the incoherent phase, working in the Chern-Simons framework, we have contributions from composite fermions as well as Chern-Simons gauge fields. The free energy is

$$
E_{i}=-T \ln Z \text {, }
$$

where the partition function $Z$ contains both compositefermion fields and Chern-Simons gauge fields of the two layers. Integrating out the composite fermions, we obtain ${ }^{11,57}$ (see Appendix A for details)

$$
Z=Z_{0} Z_{+} Z_{-},
$$

where $Z_{0}$ is the partition function for free fermions, and

$$
Z_{ \pm}=\int \mathcal{D} a_{ \pm} e^{-\int d \tau d^{2} x\left(a_{ \pm} D_{ \pm}^{-1} a_{ \pm} / 2\right)}
$$

where $a_{ \pm}$are the in-phase and out-of-phase combinations of Chern-Simons gauge fields of the two layers, and the polarizations $D_{ \pm}^{-1}$ in the Coulomb gauge have the following form

$$
D_{ \pm}^{-1}=\frac{1}{2}\left(\begin{array}{cc}
\Pi_{00}^{0} & \frac{i q}{4 \pi} \\
\frac{-i q}{4 \pi} & \Pi_{11}^{0}+\frac{2 V_{ \pm} q^{2}}{(4 \pi)^{2}}
\end{array}\right),
$$

where the index 0 and 1 denote time and transverse component, respectively,

$$
V_{ \pm}(q)=\frac{1}{2}\left[\frac{2 \pi e^{2}}{q}\left(1 \pm e^{-q d}\right)\right] F(q)
$$

is linear combinations of intralayer and interlayer Coulomb interactions, $F(q)$ is the finite-thickness form factor, ${ }^{58,59}$ and $\Pi_{00}^{0}$ and $\Pi_{11}^{0}$ are the fermion density and transverse current correlations functions, respectively,

$$
\begin{gathered}
\Pi_{00}^{0} \approx \frac{m_{*}}{\pi}\left(1+i \frac{\omega}{v_{F} q}\right), \\
\Pi_{11}^{0} \approx-\frac{q^{2}}{12 \pi m_{*}}+i \frac{2 n \omega}{k_{F} q} .
\end{gathered}
$$

$m^{*}$ is the activation mass of the composite fermions, and, as we discuss below is different from the polarization mass $m_{p}$ used in the previous section. Continuing the derivation,

$$
E_{i}=-T \ln Z=-T \ln Z_{0}-T \ln Z_{+}-T \ln Z_{-},
$$

where the free fermion part gives

$$
\frac{\partial E_{i, \text { fermion }}}{\partial T}=-\frac{\partial\left(T \ln Z_{0}\right)}{\partial T}=-\frac{2 \pi}{3} T \frac{m_{*}}{\hbar^{2}}
$$

and the gauge-field parts give ${ }^{11,60}$

$$
\frac{\partial E_{i, \pm}}{\partial T}=-\int_{0}^{\infty} \frac{\omega d \omega}{\pi T^{2}} \frac{e^{\beta \omega}}{\left(e^{\beta \omega}-1\right)^{2}} \int_{0}^{\infty} \frac{q d q}{2 \pi} \operatorname{Im} \ln \operatorname{det} D_{ \pm}^{-1} .
$$

A straightforward calculation following Ref. 11 shows that in the zero-thickness approximation [form factor $F(q)$ set to 1],

$$
\frac{\partial E_{i, \pm}}{\partial T}=-\frac{1.917}{4 \pi} \times \frac{5}{3} C_{1}^{2 / 3} T^{2 / 3}-\frac{1.645 C_{2}}{2 \pi^{2}} T \ln \frac{\omega_{0}}{T},
$$

where

$$
C_{1}=\frac{16 \pi n}{k_{F} d e^{2} / \epsilon}, \quad C_{2}=\frac{8 \pi n}{k_{F} e^{2} / \epsilon}, \quad \omega_{0}=\frac{\left(2 k_{F}\right)^{2}}{C_{2}},
$$

where $n$ is the single-layer density of composite fermions and $k_{F}=\sqrt{2 \pi n}$.

Finite thickness corrections to the form of Coulomb interaction is found to have negligible effect on the value of $\eta$, partly because it only affects the gauge-field contribution which is itself dominated by the free composite-fermion qua- 


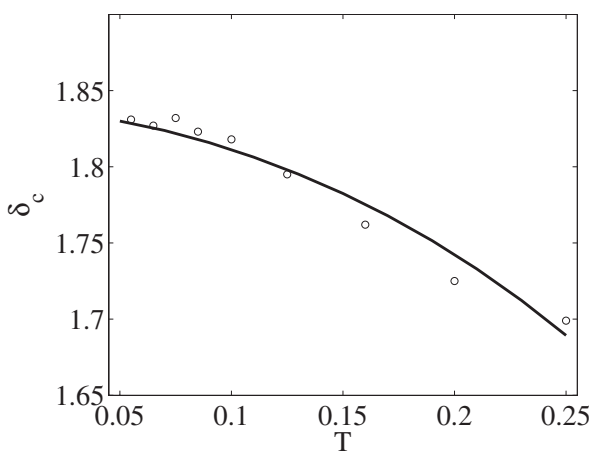

FIG. 2. The phase boundary $d / l$ vs the temperature $T$ (in kelvin) for the finite-temperature experiments. Circles are experimental results of Champagne et al. (Ref. 31) [cf. Fig. 2(c) there]. Solid line is our theoretical calculation with the fitting parameter $\eta=0.7 \times 10^{-3}$. The boundary condition in our calculation is chosen as $\delta_{c}=1.83$ when $T=50 \mathrm{mK}$.

siparticle contribution for experimentally relevant temperatures and for the choice of $m_{*}$ discussed below.

The value of the composite-fermion mass $m_{*}$ is believed to be close to the value determined by the activation gaps of fractional quantum Hall phases away from $\nu=1 / 2$. $^{11,13,15,57}$ Therefore, we use the experimental value of this activation mass determined from gap measurements in Refs. 61 and 62, which is

$$
\frac{m_{*}}{m_{e} \sqrt{B_{\perp}}}=0.2 \pm 0.02 .
$$

Note that in numerical calculations the activation mass is typically smaller than experimental value by about a factor of $2,{ }^{11,63,64}$ but it is believed that the theoretical value should approach experimental value once finite-thickness effect, disorder and Landau-level mixing are taken into account. ${ }^{64-67}$ Therefore, we feel the use of experimental value stated above is more appropriate. Also note that the polarization mass $m_{p}$ we used in the previous section is different from the mass we use here. Conceptually, within the Landau-Fermi-liquid theory, the two masses are related by $m_{p}=m_{*} /\left(1+F_{0}^{a}\right), F_{0}^{a}$ being the zeroth spin-asymmetric Landau parameter.

Using this value of the mass along with the forms of free energy in Clausius-Clapeyron Eq. (5), we get an ODE, which can be solved with the boundary condition that $\delta_{c}=1.83$ when $T=50 \mathrm{mK}$ to yield the $\delta_{c}-T$ curve plotted in Fig. 2 . To make this curve resemble the experimental result of Ref. 31 , we have set

$$
\eta=(0.7 \pm 0.2) \times 10^{-3},
$$

where the error mainly comes from the uncertainty in the value of the activation mass $m_{*}$ and also the fitting error, meaning a finite range of $\eta$ 's make the $\delta_{c}-T$ curve resemble the experimental result.

In the above calculation, we assumed that the composite Fermi liquid is spin unpolarized, and one might wonder how partial spin polarization would affect the result. Because the free fermion contribution dominates $\partial E_{i} / \partial T$ and it is proportional to the density of states of composite fermions, our results would stay the same for partially polarized composite Fermi liquid.

\section{DENSITY IMBALANCE EXPERIMENTS}

References 24 and 32 have studied the dependence of the critical $\delta_{c}=d / l$ on the density imbalance between the layers. They observed that at small imbalance, the phase boundary has a quadratic dependence on the density imbalance, and the coherent quantum Hall phase survives at higher $d / l$ with larger imbalance.

Denoting the density of the two layers $n_{1,2}$, a density imbalance between the layers,

$$
\Delta n \equiv \frac{n_{1}-n_{2}}{2}
$$

costs an energy which includes a dominating geometrical capacitance term and quantum-mechanical corrections. This is true for both phases. For the coherent phase, we follow Ref. 2 to obtain the free-energy density to be

$$
\begin{gathered}
E_{c}=\left(\frac{2 \pi e^{2} d}{\epsilon}+\beta_{m, E}\right)(\Delta n)^{2}, \\
\beta_{m, E}=\int_{0}^{\infty} \frac{q d q}{2 \pi} V^{2}(q) h(q),
\end{gathered}
$$

where $2 \pi e^{2} d / \epsilon$ is the geometrical capacitance term while $\beta_{m, E}$ is the exchange contribution which tends to offset the geometrical capacitance term. Here, $V^{z}(q)=V(q)-U(q)$, $V(q)=\frac{2 \pi e^{2}}{\epsilon q} F(q)$ is the intralayer Coulomb interaction, $F(q)$ is the finite-thickness form factor, ${ }^{58,59} U(q)=V(q) e^{-q d}$ is the interlayer Coulomb interaction, and $h(q)=-2 \pi l^{2} \exp \left(-q^{2} l^{2} / 2\right)$ is the pair distribution function of the Halperin $(1,1,1)$ wave function.

The free-energy density of the incoherent phase is (see Appendix B for details)

$$
E_{i}=\frac{(\Delta n)^{2}}{\tilde{K}-\tilde{K}^{\prime}},
$$

where

$$
\begin{gathered}
\left.\tilde{K} \equiv \frac{1}{\beta A} \lim _{\vec{q} \rightarrow 0 \omega \rightarrow 0} \lim _{1, \vec{q}, \omega} \rho_{1,-\vec{q},-\omega}\right\rangle, \\
\left.\tilde{K}^{\prime} \equiv \frac{1}{\beta A} \lim _{\vec{q} \rightarrow 0 \omega \rightarrow 0} \lim _{1, \vec{q}, \omega} \rho_{2,-\vec{q},-\omega}\right\rangle,
\end{gathered}
$$

where $\beta$ is the inverse of temperature, $A$ is the area of the sample, and $\rho_{1,2}$ are the composite-fermion density of each layer. Treating the Coulomb interaction within random-phase approximation (RPA), we obtain (see Appendix B for details)

$$
\widetilde{K}=-\widetilde{K}^{\prime}=\frac{\kappa}{2\left(1+\frac{2 \pi e^{2} d}{\epsilon} \cdot \kappa\right)},
$$

where $\kappa$ is the $\omega \rightarrow 0, q \rightarrow 0$ limit of the one-particleirreducible (1PI) density response function, namely, compressibility, of a single-layer composite Fermi liquid. Plugging Eq. (42) into Eq. (40), one obtains the energy cost of uniform density imbalance in the incoherent phase 


$$
E_{i}=\left(\frac{1}{\kappa}+\frac{2 \pi e^{2} d}{\epsilon}\right) \Delta n^{2} .
$$

From the Clausius-Clapeyron Eq. (8), the geometrical capacitance term of the two phases cancels out, and we have

$$
\eta=\frac{\kappa^{-1}-\beta_{m, E}}{\frac{d \delta_{c}}{d\left(\Delta n^{2}\right)} \frac{e^{2}}{\epsilon l^{3}}} .
$$

Since $\kappa$ is the single-layer compressibility, it is connected to the ground-state energy per area of the composite Fermi liquid $E_{G S}$ via

$$
\kappa^{-1}=\frac{\partial^{2} E_{G S}}{\partial n^{2}} .
$$

Note that our definition of the compressibility is slightly different from some literature, where $\kappa^{-1}=n^{2} \frac{\partial^{2} E_{G S}}{\partial n^{2}}$ are used instead.

Alternatively, treating the Chern-Simons interaction within RPA (see Appendix B for details), we obtain

$$
\kappa^{-1}=\kappa_{0}^{-1}-16 \pi^{2} \chi_{d}
$$

where

$$
\kappa_{0}=\frac{m_{*}}{\pi\left(1+F_{0}^{s}\right)}
$$

is the compressibility without the Chern-Simons interaction, $F_{0}^{S}$ is the zeroth Landau parameter in the spin-symmetric channel, and

$$
\chi_{d}=-\frac{1}{12 \pi m_{*}}
$$

is the Landau diamagnetic susceptibility. Therefore,

$$
E_{i}=\left(\frac{\pi}{m_{*}}+\frac{\pi F_{0}^{s}}{m_{*}}+\frac{4 \pi}{3 m_{*}}+\frac{2 \pi e^{2} d}{\epsilon}\right) \Delta n^{2} .
$$

Clearly, we can identify the four terms as free fermion contribution, exchange/correlation effect, Landau diamagnetism for Chern-Simons flux, ${ }^{32}$ and geometric capacitance term, respectively.

Although the Chern-Simons expression of $\kappa$, Eq. (49), offers valuable physical insight into its structure, the precise value of the parameters $m_{*}, \chi_{d}$, and especially $F_{0}^{s}$ are not very well understood. The best way to estimate $\kappa$ is to use its connection with ground-state energy density $E_{G S}$ of composite Fermi liquid, Eq. (45). In the zero-thickness approximation, Park et al. ${ }^{68}$ have estimated the value of $E_{G S}$ for spinunpolarized composite Fermi liquid to be

$$
E_{G S}=-0.4695 \frac{e^{2}}{\epsilon l} n,
$$

thus

$$
\kappa^{-1}=-0.4695 \times 3 \pi \frac{e^{2}}{\epsilon} l,
$$

where $n$ is the single-layer density of composite fermions and $l$ is the magnetic length.

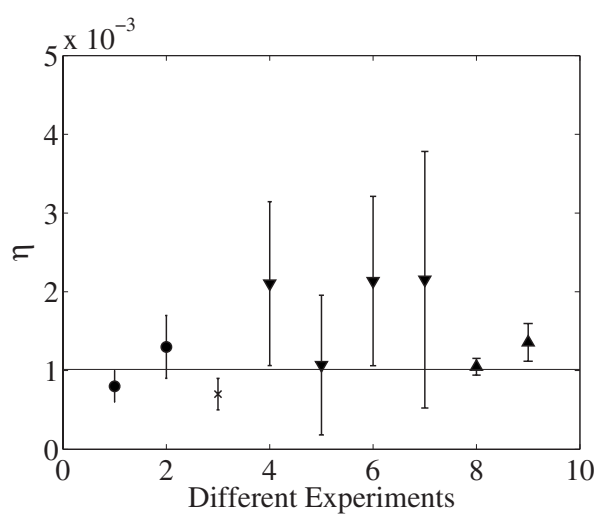

FIG. 3. Summary of the value of $\eta$ extracted from various experiments. Experiment 1: parallel field experiment of Ref. 30. Experiment 2: NMR/heat-pulse experiment of Ref. 26. Experiment 3: finite-temperature transition experiment of Ref. 31. Experiments 4-7: density imbalance experiments of Ref. 32 with $T=55,85,125$, and $200 \mathrm{mK}$. Experiments 8 and 9: density imbalance experiments of Ref. 24 with phase boundary determined by Hall drag and tunneling. To obtain this result we used the numerical result of Ref. 68 for unpolarized composite Fermi-liquid ground-state energy to estimate $\kappa^{-1}$. The horizontal line is the average value of $\eta$ weighted by inverse of error square, which is $\sim(1 \pm 0.1) \times 10^{-3}$.

Using this value of $\kappa^{-1}$ and the zero-thickness form of Coulomb interaction to calculate the coherent-phase exchange term $\beta_{m, E}$ (because the numerical result for $E_{G S}$ of the incoherent phase quoted above from Ref. 68 was also done with zero thickness), and extracting the curvature $\frac{d \delta_{c}}{d\left(\Delta n^{2}\right)}$ from experiments, we readily obtain the value of $\eta$. This result does not depend on the Chern-Simons description of composite fermions. We have plotted in Fig. 3 the values of $\eta$ extracted from density imbalance experiments as well as those determined from spin transition and finite-temperature transition experiments. The error bars for the density imbalance experiments mainly come from fitting errors.

Note that the main effect of the finite-thickness correction to the form of Coulomb interaction is to reduce the exchange terms of both phases. Since the value of $\eta$ is related to the difference between the exchange term of the two phases, we do not expect the result of $\eta$ to sensitively depend on this effect. Nevertheless, we can include it in the Chern-Simons treatment of $\kappa$. We use the activation mass $m_{*}=0.2 m_{e} \sqrt{B_{\perp}}$ estimated in Sec. III as the value of $m_{*}$, set $\chi_{d}=$ $-1 /\left(12 \pi m_{*}\right)$, and use the Hubbard approximation to estimate $F_{0}$. In the Hubbard approximation, the exchange effect is taken into account by introducing a many-body local-field factor $G(q)=q /\left(2 \sqrt{q^{2}+k_{F}^{2}}\right)$ and $F_{0}^{s}=-\frac{m_{*}}{\pi} \lim _{q \rightarrow 0} V(q) G(q)$. Thus, we obtain from Eq. (46),

$$
\kappa^{-1}=\frac{7}{3} \frac{\pi \hbar^{2}}{m_{*}}-\frac{\pi e^{2}}{\epsilon k_{F}} .
$$

Using this value of $\kappa^{-1}$ and the finite-thickness form of Coulomb interaction to calculate the coherent-phase exchange term $\beta_{m, E}$, we have calculated the values of $\eta$ from density imbalance experiments which turned out to be extremely close to the results obtained earlier in Fig. 3. 
Comments about the value of the compressibility in the composite Fermi-liquid phase are in order. First, in Ref. 69, the compressibility of a single-layer $2 \mathrm{DEG}$ at zero field was studied in detail, and it was found that aside from the wellknown density-of-states contribution and exchange contribution to the compressibility, there is a third contribution coming from the so-called Hartree band-bending effect due to the influence of the finite quantum well width on the out-ofplane direction of electron wave function. For the bilayer system studied here, we expect a similar effect on the composite Fermi-liquid compressibility $\kappa^{-1}$ in the incoherent phase and on $\beta_{m, E}$ for the coherent quantum Hall phase as well. A quantitative analysis of this effect and its impact on the density imbalance experiments is beyond the scope of this paper, and we simply note that the Hartree band-bending effect is essentially a single-particle effect, ${ }^{69}$ and therefore it will contribute equally to $\kappa^{-1}$ and $\beta_{m, E}$. To obtain the value of $\eta$ from Eq. (44), we only need the difference between $\kappa^{-1}$ and $\beta_{m, E}$, and therefore we do not expect the Hartree bandbending effect to modify our results. Second, quenched disorder acts to broaden the Landau levels and therefore adds a positive contribution to the compressibility. This could account for the close-to-zero compressibility measured by Ref. 69. Again, this effect is likely to be similar for both phases, and we do not expect disorder to affect the difference between $\kappa^{-1}$ and $\beta_{m, E}$ appreciably. Nevertheless, disorder is important in smearing the first-order transition into a continuous one (see discussion in Sec. V).

We assumed that the composite Fermi liquid is unpolarized above but again we do not expect partial polarization to affect our results strongly. For Eq. (51), Park et al. ${ }^{68}$ also reported the ground-state energy for polarized composite Fermi liquid to be very close to the unpolarized one quoted above,

$$
E_{\text {polarized }}=-0.4656 \frac{e^{2}}{\epsilon l} n
$$

and, therefore, our results would also stay very close. In the Chern-Simons treatment, Eqs. (49) and (52), since the Chern-Simons fields couple to both spins and the density and current response function stays the same for partially polarized and unpolarized composite Fermi liquids, our calculation also remains valid (see Appendix B).

\section{SUMMARY AND DISCUSSION}

To summarize, we derived the Clausius-Clapeyron relations [Eqs. (3), (5), and (8)] for the phase transition tuned by $d / l$ in bilayer $\nu_{t o t}=1$ quantum Hall system, assuming that it is a first-order transition between spin-polarized coherent quantum Hall state and spin partially polarized compositefermion Fermi-liquid state. In Sec. II, we studied the changes in phase boundary $(d / l)_{c}$ when the magnetic field coupled to spin is changed by either NMR/heat pulse or parallel magnetic field. The phase boundary as a function of temperature was studied in Sec. III. The temperature dependence of free energy in the coherent quantum Hall phase is dominated by the linearly, dispersing Goldstone mode while the incoherent composite Fermi-liquid phase has contributions from both fermions and gauge fields. In Sec. IV, we investigated the changes in phase boundary when there is density imbalance between the two layers. We use the result of Ref. 2 for the free-energy cost of density imbalance in the coherent quantum Hall phase. The free energy for the incoherent phase is shown to be connected to the compressibility of single-layer composite Fermi liquid.

Our main goal was to check the consistency of the Clausius-Clapeyron relation with the observed transition. Each experiment which observes the change in $(d / \ell)$ due to changing another parameter in the system indicates a value for $\eta$, as defined in Eq. (4); all values should agree.

In Fig. 3, we have plotted the values of $\eta$ determined from spin transition, finite-temperature transition, and density imbalance transition experiments. The horizontal line is the average value of $\eta$ weighted by inverse of error square, i.e., the maximum-likelihood estimator of $\eta$. One can see that, indeed, all nine values of $\eta$ extracted from various experiments roughly lie in the range $1 \times 10^{-3}-2 \times 10^{-3}$, and the weighted average value of $\eta=(1 \pm 0.1) \times 10^{-3}$ is roughly within all the error bars. Our analysis, therefore, confirms the consistency for the scenario of a direct first-order phase transition between coherent quantum Hall phase and incoherent composite Fermi-liquid phase. Furthermore, the analysis provides a unified framework within which we can understand the observed phase boundaries for several distinct experiments.

In Sec. IV, we also worked in the Chern-Simons description of composite fermions [i.e., Eq. (52)] in addition to our treatment [i.e., Eq. (51)] using the numerical results of Ref. 68 , and we obtained extremely similar results. Stepping back a little from that analysis with the Chern-Simons treatment, one can pretend ignorance of any knowledge of the parameters including the effective mass $m_{*}$ and the exchange contribution to $\kappa^{-1}$, and ask what values of them would give good agreement between the values of $\eta$ extracted from experiments. We have plotted the standard deviation of $\eta$ extracted from various experiments divided by the their average value in Fig. 4 as a function of the composite-fermion mass (in units of $m_{e} \sqrt{B_{\perp}}$ ) and the exchange contribution to $\kappa^{-1}$, which is $F_{0}^{s} \pi / m_{*}$ (in units of $e^{2} l / \epsilon$ ). The finite-thickness form of the Coulomb interaction is used in calculating the coherent-phase exchange term when producing this plot. Gray color denotes the region where at least one of the $\eta$ 's becomes negative, thus unphysical, while dark blue denotes parameter regimes which give rise to good agreement among $\eta$ 's extracted from different experiments. The horizontal line denotes the Hubbard approximation to the exchange effect $(\sqrt{2} \pi)$, while he vertical line denotes the experimental value of the activation mass $m_{*} \approx 0.2 m_{e} \sqrt{B_{\perp}}$.

We have not explicitly discuss the role of disorder, which is always present in the samples. Disorder will bring spatial fluctuations into some variables in the Clausius-Clapeyron equations we have derived, and therefore smear the firstorder transition into a continuous one, as observed in experiments. Roughly speaking, the analysis we have performed in this work applies to the spatially averaged quantities. For example, with disorder, the RHS of the Clausius-Clapeyron equation for spin transitions, Eq. (15), will acquire spatial dependence most likely through a spatially fluctuating spin susceptibility $\chi$, 


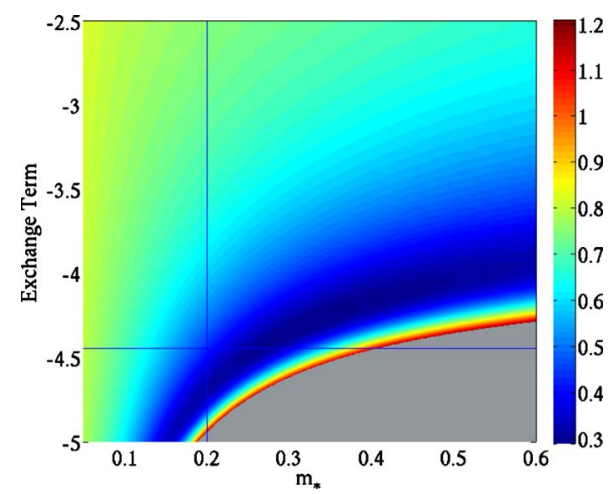

FIG. 4. (Color online) Standard deviation of $\eta$ among various experiments divided by their average value (which measures the goodness of agreement between $\eta$ 's) within the Chern-Simons framework as a function of the composite-fermion mass and the exchange contribution to $\kappa^{-1}$ [see Eq. (52)]. Horizontal axis: composite-fermion mass in units of $m_{e} \sqrt{B_{\perp}}, m_{e}$ being the vacuum electron mass, $B_{\perp}$ is in units of tesla. Vertical axis: exchange contribution to $\kappa^{-1}$, which is $F_{0}^{s} \pi / m_{*}$ (in units of $e^{2} l / \epsilon, l$ being the magnetic length). Gray color denotes the region where at least one of the $\eta$ 's becomes negative, thus unphysical. The horizontal line denotes the Hubbard approximation to the exchange effect ( $-\sqrt{2} \pi)$. The vertical line denotes the experimental value of the activation mass $m_{*} \approx 0.2 m_{e} \sqrt{B_{\perp}}$, which is the value of compositefermion mass we used in calculations for Figs. 2 and 3.

$$
\frac{d \delta_{c}(\vec{x})}{d B_{t o t}}=\frac{-2 \chi(\vec{x}) B_{t o t}+\frac{e g \mu_{B} B_{\perp}}{4 \pi \hbar}}{\eta \frac{e^{2}}{\epsilon l^{3}}} .
$$

Thus, one can take the spatial average of both sides and study how the averaged critical $\delta_{c}$ changes with $B_{t o t}$, as we did in this work. Furthermore, one can also take the standard deviation of both sides of Eq. (54), and conclude that the width of the phase transition, which is the standard deviation of $\delta_{c}$, grows with $B_{\text {tot }}$ assuming the standard deviation of $\chi(\vec{x})$ does not change appreciably with $B_{\text {tot }}$. One can also study the finite-temperature transition in a similar way. Because there the free fermion term, Eq. (33), dominates, one can conclude that the transition becomes wider at higher temperature, if one assumes the composite-fermion mass $m_{*}$ has some temperature-independent spatial variation. This is in accord with the experimental observation of Ref. 31.

A major question which is not directly addressed in our analysis is the possibility of a continuous quantum crossover between the coherent and incoherent phases (see, e.g., Refs. 47 and 48). If indeed no real thermodynamic singularity exists even in the clean case, then there is no reason for the Clausius-Clapeyron relations to hold as well as we find they do. Nonetheless, there is also no contradiction in them holding where no first-order transition exists. In this case, however, we can draw the conclusion that the crossover region between the two phases must be very narrow, such that it approximates a smeared thermodynamic singularity (just as disorder would widen a thermodynamic singularity) and therefore follows the Clausius-Clapeyron relations we pre- sented here for the unmixed phases. In other words, a good agreement with the relations indicates that already at regions in parameter space close to the transition, the thermodynamic functions of the pure coherent and pure incoherent phases apply, and they indicate a smeared phase-transition line.

Additional outstanding questions which we did not address but are noteworthy are as follows. First, a thermodynamic phase transition between the coherent and incoherent phases does not have to be first order at high Zeeman fields when both phases are spin polarized; a second-order phase transition is not ruled out a priori. Future experiments should clarify this issue (see the recent experiments of Refs. 34 and 35). In addition, for the density imbalance transitions, we have mainly focused on the regime of small imbalance, while the experiments of Ref. 32 have studied the case of large imbalance, e.g., $\Delta \nu=\nu_{1}-\nu_{2} \leq 0.4$. The interlayer incoherent phase in that regime could be two decoupled singlelayer fractional quantum Hall phase. It would be very interesting to see if a similar Clausius-Clapeyron equation can describe the phase transition in that case. Finally, although our assumption, Eq. (4), is very natural on qualitative ground, a microscopic derivation of this quantity would be very useful.

\section{ACKNOWLEDGMENTS}

It is a pleasure to acknowledge useful conversations with J. Alicea, A. Champagne, H. Fertig, A. D. K Finck, A. H. MacDonald, G. Murthy, F. von Oppen, E. Rezayi, S. Simon, and I. Spielman. We thank K. Muraki for providing us the experimental data shown in Fig. 1. We are grateful for support from the research corporation, the Packard Foundation, and the Sloan foundation (G.R.), the Israel-U.S. Binational Science Foundation, the Minerva Foundation, Microsoft Station Q (A.S.), and NSF under Grant No. DMR-0552270 (J.P.E.).

\section{APPENDIX A: DERIVATION OF THE TEMPERATURE DEPENDENCE OF THE COMPOSITE FERMI- LIQUID FREE ENERGY}

Within the Chern-Simons description of the compositefermion Fermi liquid at $\nu=1 / 2$, we have the following partition function of the system:

$$
Z=\int \mathcal{D} a_{1} \mathcal{D} a_{2} \mathcal{D} \psi_{1 \sigma} \mathcal{D} \psi_{2 \sigma} e^{-\int d \tau d^{2} x \mathcal{L}},
$$

where

$$
\begin{aligned}
\mathcal{L}= & \sum_{n=1,2}\left\{\psi_{n \sigma}^{\dagger}\left(\partial_{\tau}-\mu_{n}-i a_{n, 0}\right) \psi_{n \sigma}-\frac{i}{8 \pi} a_{n \mu} \epsilon^{\mu \nu \lambda} \partial_{\nu} a_{n \lambda}\right. \\
& +\frac{1}{2 m} \psi_{n \sigma^{\dagger}}^{\dagger}\left(-i \nabla-\vec{a}_{n}\right)^{2} \psi_{n \sigma}+\frac{1}{2} \int d^{2} x^{\prime} \psi_{n \sigma}^{\dagger}(x) \psi_{n \sigma}(x) V(x \\
& \left.\left.-x^{\prime}\right) \psi_{n \sigma^{\prime}}^{\dagger}\left(x^{\prime}\right) \psi_{n \sigma^{\prime}}\left(x^{\prime}\right)\right\}+\int d^{2} x^{\prime} \psi_{1 \sigma}^{\dagger}(x) \psi_{1 \sigma}(x) U(x \\
& \left.-x^{\prime}\right) \psi_{2 \sigma^{\prime}}^{\dagger}\left(x^{\prime}\right) \psi_{2 \sigma^{\prime}}\left(x^{\prime}\right),
\end{aligned}
$$


where $\psi_{n \sigma}$ is the composite-fermion fields in the $n$th layer with spin $\sigma, V$ and $U$ are the intralayer and interlayer Coulomb interaction, respectively. Here, $a_{n \mu}$ are the fluctuations of the Chern-Simons gauge fields in the $n$th layer from its saddle-point value which cancels the external magnetic field exactly and $\mu=0,1,2$ are the time and two spatial coordinates, respectively. Integrating out $a_{n, 0}$, one obtains the expected constraints

$$
\nabla \times \vec{a}_{n}=4 \pi \psi_{n \sigma}^{\dagger} \psi_{n \sigma} .
$$

Following Ref. 11, we make use of this constraint and replace $\psi_{n \sigma}^{\dagger} \psi_{n \sigma}$ in Coulomb interaction terms by $\nabla \times \vec{a}_{n} /(4 \pi)$. Next, we define

$$
\begin{gathered}
a_{ \pm \mu}=a_{1 \mu} \pm a_{2 \mu}, \\
V_{ \pm}=\frac{V \pm U}{2},
\end{gathered}
$$

and reorganize $\mathcal{L}$ as

$$
\begin{aligned}
& \mathcal{L}=\mathcal{L}_{f}+\mathcal{L}_{C S}, \mathcal{L}_{f}= \\
& \psi_{1 \sigma}^{\dagger}\left(\partial_{\tau}-\mu_{1}-i \frac{a_{+0}+a_{-0}}{2}\right) \psi_{1 \sigma}+\psi_{2 \sigma}^{\dagger}\left(\partial_{\tau}-\mu_{2}-i \frac{a_{+0}-a_{-0}}{2}\right) \psi_{2 \sigma}+\psi_{1 \sigma}^{\dagger} \frac{\left[-i \nabla-\left(\vec{a}_{+}+\vec{a}_{-}\right) / 2\right]^{2}}{2 m} \psi_{1 \sigma} \\
&+\psi_{2 \sigma}^{\dagger} \frac{\left[-i \nabla-\left(\vec{a}_{+}-\vec{a}_{-}\right) / 2\right]^{2}}{2 m} \psi_{2 \sigma}, \mathcal{L}_{C S}=-\frac{i}{16 \pi} a_{+\mu} \epsilon^{\mu \nu \lambda} \partial_{\nu} a_{+\lambda}-\frac{i}{16 \pi} a_{-\mu} \epsilon^{\mu \nu \lambda} \partial_{\nu} a_{-\lambda}+\frac{1}{2} \frac{1}{(4 \pi)^{2}} \int d^{2} x^{\prime}\left[\nabla \times \vec{a}_{+}(x)\right] V_{+}\left(x-x^{\prime}\right)[\nabla \\
&\left.\times \vec{a}_{+}\left(x^{\prime}\right)\right]+\frac{1}{2} \frac{1}{(4 \pi)^{2}} \int d^{2} x^{\prime}\left[\nabla \times \vec{a}_{-}(x)\right] V_{-}\left(x-x^{\prime}\right)\left[\nabla \times \vec{a}_{-}\left(x^{\prime}\right)\right] .
\end{aligned}
$$

Denoting the free fermion partition function to be

$$
Z_{0}=\int \mathcal{D} \psi_{1 \sigma} \mathcal{D} \psi_{2 \sigma} \exp \left[-\int d \tau d^{2} x \mathcal{L}_{f}\left(a_{ \pm}=0\right)\right],
$$

and following standard methods ${ }^{11}$ to integrate out compositefermion fields $\psi_{n \sigma}$, we obtain

$$
Z=Z_{0} Z_{+} Z_{-} \text {, }
$$

where $Z_{0}$ is the partition function for free fermions, and

$$
Z_{ \pm}=\int \mathcal{D} a_{ \pm} e^{-\int d \tau d^{2} x\left(a_{ \pm} D_{ \pm}^{-1} a_{ \pm} / 2\right)} .
$$

In Coulomb gauge, one can treat the polarizations $D_{ \pm}^{-1}$ as 2 $\times 2$ matrices with index 0 and 1 to be the time and transverse component, respectively. Thus, $D_{ \pm}^{-1}$ take the following form:

$$
D_{ \pm}^{-1}=\frac{1}{2}\left(\begin{array}{cc}
\Pi_{00}^{0} & \frac{i q}{4 \pi} \\
\frac{-i q}{4 \pi} & \Pi_{11}^{0}+\frac{2 V_{ \pm} q^{2}}{(4 \pi)^{2}}
\end{array}\right)
$$

where $\Pi_{00}^{0}$ and $\Pi_{11}^{0}$ are the density and transverse current correlation functions of free fermions resulted from integrating out composite-fermion fields. Thus, the free energy is given by

$$
E_{i}=-T \ln Z=-T \ln Z_{0}-T \ln Z_{+}-T \ln Z_{-}
$$

and the rest of the steps are given in Sec. III.

\section{APPENDIX B: DERIVATION OF THE FREE ENERGY FOR DENSITY IMBALANCE IN COMPOSITE FERMI- LIQUID PHASE}

Starting from action, Eq. (A1), or any other action for composite fermions, we integrate out all fluctuating fields and obtain

$$
\begin{aligned}
Z= & \exp \left\{\frac { 1 } { 2 \beta A } \sum _ { \vec { q } , \omega } \left[K_{\vec{q}, \omega} \phi_{1, \vec{q}, \omega} \phi_{1,-\vec{q},-\omega}+K_{\vec{q}, \omega} \phi_{2, \vec{q}, \omega} \phi_{2,-\vec{q},-\omega}\right.\right. \\
& \left.\left.+2 K_{\vec{q}, \omega}^{\prime} \phi_{1, \vec{q}, \omega} \phi_{2,-\vec{q},-\omega}\right]\right\},
\end{aligned}
$$

where

$$
\begin{gathered}
K_{\vec{q}, \omega}=\frac{1}{\beta A}\left\langle\rho_{1, \vec{q}, \omega} \rho_{1,-\vec{q},-\omega}\right\rangle=\frac{1}{\beta A}\left\langle\rho_{2, \vec{q}, \omega} \rho_{2,-\vec{q},-\omega}\right\rangle, \\
K_{\vec{q}, \omega}^{\prime}=\frac{1}{\beta A}\left\langle\rho_{1, \vec{q}, \omega} \rho_{2,-\vec{q},-\omega}\right\rangle,
\end{gathered}
$$

where $\rho_{j}$ is the composite-fermion density of the $j$ th layer, $\beta$ is the inverse of the temperature, $A$ is the area of the sample, and $\phi_{j, \vec{q}, \omega}$ is the Fourier-transformed potential in the $j$ th layer. For a constant potential $\phi_{j}(j=1,2)$, we have

$$
\phi_{j, \vec{q}, \omega}=\phi_{j} \cdot \beta A \delta_{\vec{q}, 0} \delta_{\omega, 0},
$$

and the grand potential $\Omega$ is

$$
\Omega=-T \ln Z=-\frac{A}{2}\left(\tilde{K} \phi_{1}^{2}+\tilde{K} \phi_{2}^{2}+2 \tilde{K}^{\prime} \phi_{1} \phi_{2}\right),
$$

where 


$$
\tilde{K} \equiv \lim _{\vec{q} \rightarrow 0 \omega \rightarrow 0} \lim _{\vec{q}, \omega}, \quad \tilde{K}^{\prime} \equiv \lim _{\vec{q} \rightarrow 0 \omega \rightarrow 0} \lim _{\vec{q}, \omega}^{\prime} .
$$

The density in each layer is

$$
\begin{aligned}
& n_{1}=-\frac{1}{A} \frac{\partial \Omega}{\partial \phi_{1}}=\tilde{K} \phi_{1}+\tilde{K}^{\prime} \phi_{2}, \\
& n_{2}=-\frac{1}{A} \frac{\partial \Omega}{\partial \phi_{2}}=\tilde{K} \phi_{2}+\tilde{K}^{\prime} \phi_{1} .
\end{aligned}
$$

Finally, the free energy is obtained via a Legendre transformation

$$
F=\Omega+\phi_{1} n_{1} A+\phi_{2} n_{2} A=\frac{A}{4}\left[\frac{\left(n_{1}-n_{2}\right)^{2}}{\tilde{K}-\tilde{K}^{\prime}}+\frac{\left(n_{1}+n_{2}\right)^{2}}{\tilde{K}+\tilde{K}^{\prime}}\right] .
$$

Within the RPA treatment of the Coulomb interaction, the full density response function $K$ is related to its 1PI counterpart $\Pi$ (which neglects the long-range Coulomb interaction) by

$$
K^{-1}=\Pi^{-1}+\tilde{V}
$$

where $K, \Pi$, and $\widetilde{V}$ are $2 \times 2$ matrices in the layer-index space,

$$
\tilde{V}=\left(\begin{array}{cc}
V & U \\
U & V
\end{array}\right), \quad \Pi=\left(\begin{array}{cc}
\Pi_{00} & 0 \\
0 & \Pi_{00}
\end{array}\right) .
$$

Here, $V$ and $U$ are intralayer and interlayer Coulomb interaction potential, respectively, and $\Pi_{00}$ in the static uniform limit gives the single-layer compressibility $\kappa$,

$$
\kappa \equiv \lim _{q \rightarrow 0 \omega \rightarrow 0} \lim _{00}
$$

Solving Eq. (B8), we have

$$
\begin{gathered}
K_{11}=K_{22}=\frac{\Pi_{00}\left(1+\Pi_{00} V\right)}{\left(1+\Pi_{00} V\right)^{2}-\Pi_{00}^{2} U^{2}}, \\
K_{12}=\frac{-\left(\Pi_{00}\right)^{2} U}{\left(1+\Pi_{00} V\right)^{2}-\Pi_{00}^{2} U^{2}} .
\end{gathered}
$$

Given the form of Coulomb interactions

$$
V(q)=\frac{2 \pi e^{2}}{q} F(q), \quad U(q)=V(q) e^{-q d},
$$

and the fact that the finite-thickness form factor $F(q) \rightarrow 1$ as $q \rightarrow 0$, in the limit $\omega \rightarrow 0$ and $q \rightarrow 0$, the denominators of $K_{11}$, $K_{22}$, and $K_{12}$ become

$$
\begin{aligned}
\left(1+\Pi_{00} V\right)^{2}-\left(\Pi_{00}\right)^{2} U^{2} \rightarrow & \frac{4 \pi e^{2} \kappa}{\epsilon q}\left(1+\frac{2 \pi e^{2} \kappa d}{\epsilon}\right), \\
& \text { as } \omega \rightarrow 0, q \rightarrow 0 .
\end{aligned}
$$

Therefore in this limit

$$
\begin{gathered}
\tilde{K} \equiv \lim _{q \rightarrow 0 \omega \rightarrow 0} \lim _{11}=\frac{\kappa}{2\left(1+2 \pi e^{2} \kappa d / \epsilon\right)}, \\
\tilde{K}^{\prime} \equiv \lim _{q \rightarrow 0 \omega \rightarrow 0} \lim _{12}=-\frac{\kappa}{2\left(1+2 \pi e^{2} \kappa d / \epsilon\right)},
\end{gathered}
$$

and the imbalance part of the free-energy density is

$$
E_{i}=\lim _{\vec{q} \rightarrow 0 \omega \rightarrow 0} \lim \frac{\Delta n^{2}}{\tilde{K}-\tilde{K}^{\prime}}=\left(\frac{1}{\kappa}+\frac{2 \pi e^{2} d}{\epsilon}\right) \Delta n^{2},
$$

as shown in Sec. IV. This result does not depend on the Chern-Simons description of composite fermions. Note also that the total compressibility $\tilde{K}+\tilde{K}^{\prime}$ vanishes linearly in $q$ as $q \rightarrow 0$ due to the long-range nature of the Coulomb interaction, similar to the single-layer case as analyzed by Halperin et al. ${ }^{11}$

To calculate the single-layer compressibility $\kappa$ within the Chern-Simons framework, we have the following RPA equation:

$$
(\Pi)^{-1}=\left(\Pi^{0}\right)^{-1}+C,
$$

where $C$ is the propagator of the Cherns-Simons field and $\Pi^{0}$ is the correlation functions without the Chern-Simons interaction. We work in the Coulomb gauge and treat $\Pi, \Pi^{0}$, and $C$ as $2 \times 2$ matrices in the space of density and transverse current. In the static and long-wavelength limit, we have

$$
\Pi^{0}=\left(\begin{array}{cc}
\kappa_{0} & 0 \\
0 & \chi_{d} q^{2}
\end{array}\right), \quad C=\frac{4 \pi}{q}\left(\begin{array}{cc}
0 & i \\
-i & 0
\end{array}\right),
$$

where $\kappa_{0}=m_{*} /\left[\pi \hbar^{2}\left(1+F_{0}^{s}\right)\right]$ is the density response function neglecting Chern-Simons interaction and $\chi_{d}$ is the Landau diamagnetic susceptibility. Hence,

$$
\kappa^{-1}=\kappa_{0}^{-1}-16 \pi^{2} \chi_{d},
$$

as shown in Sec. IV. Note that these results are the same for unpolarized and partially polarized composite Fermi liquids, because Eq. (B15) is valid in any case since Chern-Simons fields couple to both spins, and the value of $\kappa_{0}$ and $\chi_{d}$ in Eq. (B16) stays the same for partially polarized composite Fermi liquid. The value of $F_{0}^{s}$ in the Hubbard approximation treatment is also roughly the same for partially polarized and unpolarized composite Fermi liquids.
${ }^{1}$ X. G. Wen and A. Zee, Phys. Rev. B 47, 2265 (1993).
${ }^{2}$ K. Moon, H. Mori, K. Yang, S. M. Girvin, A. H. MacDonald, L. Zheng, D. Yoshioka, and S.-C. Zhang, Phys. Rev. B 51, 5138 (1995).
${ }^{3}$ J. P. Eisenstein and A. H. MacDonald, Nature (London) 432, 691 (2004).

${ }^{4}$ I. B. Spielman, J. P. Eisenstein, L. N. Pfeiffer, and K. W. West, Phys. Rev. Lett. 84, 5808 (2000). 
${ }^{5}$ I. B. Spielman, J. P. Eisenstein, L. N. Pfeiffer, and K. W. West, Phys. Rev. Lett. 87, 036803 (2001).

${ }^{6}$ M. Kellogg, I. B. Spielman, J. P. Eisenstein, L. N. Pfeiffer, and K. W. West, Phys. Rev. Lett. 88, 126804 (2002).

${ }^{7}$ M. Kellogg, J. P. Eisenstein, L. N. Pfeiffer, and K. W. West, Phys. Rev. Lett. 93, 036801 (2004).

${ }^{8}$ A. Stern, S. M. Girvin, A. H. MacDonald, and N. Ma, Phys. Rev. Lett. 86, 1829 (2001).

${ }^{9}$ Y. N. Joglekar and A. H. MacDonald, Phys. Rev. Lett. 87, 196802 (2001).

${ }^{10}$ H. A. Fertig and G. Murthy, Phys. Rev. Lett. 95, 156802 (2005).

${ }^{11}$ B. I. Halperin, P. A. Lee, and N. Read, Phys. Rev. B 47, 7312 (1993).

${ }^{12}$ S. H. Simon and B. I. Halperin, Phys. Rev. B 48, 17368 (1993).

${ }^{13}$ A. Stern and B. I. Halperin, Phys. Rev. B 52, 5890 (1995).

${ }^{14}$ S. H. Simon, A. Stern, and B. I. Halperin, Phys. Rev. B 54, R11114 (1996).

${ }^{15} \mathrm{~S}$. H. Simon, in Composite Fermions, edited by O. Heinonen (World Scientific, Singapore, 1998).

${ }^{16}$ R. Shankar and G. Murthy, Phys. Rev. Lett. 79, 4437 (1997).

${ }^{17}$ N. Read, Phys. Rev. B 58, 16262 (1998).

${ }^{18}$ D.-H. Lee, Phys. Rev. Lett. 80, 4745 (1998).

${ }^{19}$ A. Stern, B. I. Halperin, F. von Oppen, and S. H. Simon, Phys. Rev. B 59, 12547 (1999).

${ }^{20}$ R. Shankar, Phys. Rev. B 63, 085322 (2001).

${ }^{21}$ G. Murthy and R. Shankar, Rev. Mod. Phys. 75, 1101 (2003).

${ }^{22}$ M. Kellogg, J. P. Eisenstein, L. N. Pfeiffer, and K. W. West, Phys. Rev. Lett. 90, 246801 (2003).

${ }^{23}$ E. Tutuc, S. Melinte, E. P. De Poortere, R. Pillarisetty, and M. Shayegan, Phys. Rev. Lett. 91, 076802 (2003).

${ }^{24}$ I. B. Spielman, M. Kellogg, J. P. Eisenstein, L. N. Pfeiffer, and K. W. West, Phys. Rev. B 70, 081303(R) (2004).

${ }^{25}$ W. R. Clarke, A. P. Micolich, A. R. Hamilton, M. Y. Simmons, C. B. Hanna, J. R. Rodriguez, M. Pepper, and D. A. Ritchie, Phys. Rev. B 71, 081304(R) (2005).

${ }^{26}$ I. B. Spielman, L. A. Tracy, J. P. Eisenstein, L. N. Pfeiffer, and K. W. West, Phys. Rev. Lett. 94, 076803 (2005).

${ }^{27}$ N. Kumada, K. Muraki, K. Hashimoto, and Y. Hirayama, Phys. Rev. Lett. 94, 096802 (2005).

${ }^{28}$ S. Luin, V. Pellegrini, A. Pinczuk, B. S. Dennis, L. N. Pfeiffer, and K. W. West, Phys. Rev. Lett. 94, 146804 (2005).

${ }^{29}$ L. A. Tracy, J. P. Eisenstein, L. N. Pfeiffer, and K. W. West, Phys. Rev. B 73, 121306(R) (2006).

${ }^{30}$ P. Giudici, K. Muraki, N. Kumada, Y. Hirayama, and T. Fujisawa, Phys. Rev. Lett. 100, 106803 (2008).

${ }^{31}$ A. R. Champagne, J. P. Eisenstein, L. N. Pfeiffer, and K. W. West, Phys. Rev. Lett. 100, 096801 (2008).

${ }^{32}$ A. R. Champagne, A. D. K. Finck, J. P. Eisenstein, L. N. Pfeiffer, and K. W. West, Phys. Rev. B 78, 205310 (2008).

${ }^{33}$ B. Karmakar, V. Pellegrini, A. Pinczuk, L. N. Pfeiffer, and K. W. West, Phys. Rev. Lett. 102, 036802 (2009).

${ }^{34}$ A. D. K. Finck, J. P. Eisenstein, L. N. Pfeiffer, and K. W. West, Phys. Rev. Lett. 104, 016801 (2010).

${ }^{35}$ P. Giudici, K. Muraki, N. Kumada, and T. Fujisawa, Phys. Rev. Lett. 104, 056802 (2010).

${ }^{36}$ R. Côté, L. Brey, and A. H. MacDonald, Phys. Rev. B 46, 10239 (1992).

${ }^{37}$ L. Zheng and H. A. Fertig, Phys. Rev. B 52, 12282 (1995).

${ }^{38}$ S. Narasimhan and T.-L. Ho, Phys. Rev. B 52, 12291 (1995).
${ }^{39}$ N. E. Bonesteel, I. A. McDonald, and C. Nayak, Phys. Rev. Lett. 77, 3009 (1996).

${ }^{40}$ Y. B. Kim, C. Nayak, E. Demler, N. Read, and S. Das Sarma, Phys. Rev. B 63, 205315 (2001).

${ }^{41}$ E. Demler, C. Nayak, and S. Das Sarma, Phys. Rev. Lett. 86, 1853 (2001).

${ }^{42}$ J. Schliemann, S. M. Girvin, and A. H. MacDonald, Phys. Rev. Lett. 86, 1849 (2001).

${ }^{43}$ A. Stern and B. I. Halperin, Phys. Rev. Lett. 88, 106801 (2002).

${ }^{44}$ S. H. Simon, E. H. Rezayi, and M. V. Milovanovic, Phys. Rev. Lett. 91, 046803 (2003).

${ }^{45}$ N. Shibata and D. Yoshioka, J. Phys. Soc. Jpn. 75, 043712 (2006).

${ }^{46}$ J. Ye and L. Jiang, Phys. Rev. Lett. 98, 236802 (2007).

${ }^{47}$ G. Möller, S. H. Simon, and E. H. Rezayi, Phys. Rev. Lett. 101, 176803 (2008).

${ }^{48}$ G. Möller and S. H. Simon, Phys. Rev. B 77, 075319 (2008).

${ }^{49}$ M. V. Milovanović and Z. Papić, Phys. Rev. B 79, 115319 (2009).

${ }^{50}$ L. A. Tracy, J. P. Eisenstein, L. N. Pfeiffer, and K. W. West, Phys. Rev. Lett. 98, 086801 (2007).

${ }^{51}$ Y. Q. Li, V. Umansky, K. von Klitzing, and J. H. Smet, Phys. Rev. Lett. 102, 046803 (2009).

${ }^{52}$ K. Park and J. K. Jain, Phys. Rev. Lett. 80, 4237 (1998).

${ }^{53}$ S. Melinte, N. Freytag, M. Horvatić, C. Berthier, L. P. Lévy, V. Bayot, and M. Shayegan, Phys. Rev. Lett. 84, 354 (2000).

${ }^{54}$ N. Freytag, M. Horvatić, C. Berthier, M. Shayegan, and L. P. Lévy, Phys. Rev. Lett. 89, 246804 (2002).

${ }^{55}$ I. V. Kukushkin, K. v. Klitzing, and K. Eberl, Phys. Rev. Lett. 82, 3665 (1999).

${ }^{56}$ Since the gapless Goldstone mode contribution to $\partial E / \partial T$ is smaller than $2 \%$ of the composite-fermion contribution, even if gap of the topological excitations is comparable to the temperature and therefore the topological excitation contribution to $\partial E / \partial T$ is comparable to that of the gapless Goldstone mode, the composite-fermion contribution would still dominate $\partial E / \partial T$ and our analysis is not affected.

${ }^{57}$ Y. B. Kim and P. A. Lee, Phys. Rev. B 54, 2715 (1996).

${ }^{58}$ P. J. Price, Phys. Rev. B 30, 2234 (1984).

${ }^{59}$ A. Gold, Phys. Rev. B 35, 723 (1987).

${ }^{60}$ X. G. Wen, Quantum Field Theory of Many-body Systems: From the Origin of Sound to an Origin of Light and Electrons (Oxford University Press, New York, 2007).

${ }^{61}$ R. R. Du, H. L. Stormer, D. C. Tsui, L. N. Pfeiffer, and K. W. West, Phys. Rev. Lett. 70, 2944 (1993).

${ }^{62}$ R. R. Du, H. L. Stormer, D. C. Tsui, A. S. Yeh, L. N. Pfeiffer, and K. W. West, Phys. Rev. Lett. 73, 3274 (1994).

${ }^{63}$ R. Morf and N. d'Ambrumenil, Phys. Rev. Lett. 74, 5116 (1995).

${ }^{64}$ K. Park and J. K. Jain, Phys. Rev. Lett. 81, 4200 (1998).

${ }^{65}$ D. Yoshioka, J. Phys. Soc. Jpn. 55, 885 (1986).

${ }^{66}$ R. H. Morf, Phys. Rev. Lett. 83, 1485 (1999).

${ }^{67}$ K. Park, N. Meskini, and J. K. Jain, J. Phys.: Condens. Matter 11, 7283 (1999).

${ }^{68}$ K. Park, V. Melik-Alaverdian, N. E. Bonesteel, and J. K. Jain, Phys. Rev. B 58, R10167 (1998).

${ }^{69}$ J. P. Eisenstein, L. N. Pfeiffer, and K. W. West, Phys. Rev. B 50, 1760 (1994). 